\title{
Occupational Stress, Psychological Distress, Physical Symptoms, and their Interrelationships among Frontline Nurses Treating Patients with COVID-19
}

\section{Tomoe Nishihara ( $\sim$ kamonomoe@gmail.com )}

National Hospital Organization: Dokuritsu Gyosei Hojin Kokuritsu Byoin Kiko https://orcid.org/00000002-2013-9275

Kazufumi Yoshihara

Kyushu University: Kyushu Daigaku

Ayako Ohashi

Kyushu University: Kyushu Daigaku

\section{Mika Kuroiwa}

National Hospital Organization: Dokuritsu Gyosei Hojin Kokuritsu Byoin Kiko Nobuyuki Sudo

Kyushu University: Kyushu Daigaku

\section{Research Article}

Keywords: COVID-19,frontline nurse,occupationalstress,physical symptoms, psychologicaldistress,increased workload, pain, insomnia

Posted Date: July 23rd, 2021

DOl: https://doi.org/10.21203/rs.3.rs-718031/v1

License: (c) (i) This work is licensed under a Creative Commons Attribution 4.0 International License. Read Full License 


\section{Abstract}

Background: The COVID-19 pandemic has presented mental health challenges to healthcare workers. Because of the urgency of the situation, this study was done to determine the amount of COVID-19related occupational stress, psychosomatic symptoms, psychological distress, and their correlations among frontline nurses during and after the peak of the outbreak in Japan.

Methods: Sixteen frontline nurses, aged 25-52 years, working in a ward with COVID-19 patients participated. Two months after the peak of the first wave of the COVID-19 outbreak in Japan, the COVID19-related occupational stress scale (COS, questionnaire items: fear of infection, increased workload) and physical symptom scale (PS, questionnaire items: gastrointestinal symptoms, pain, appetite loss, insomnia) were assessed. The degree of general psychological distress was evaluated using the 6-item Kessler Scale (K6). At the same time, the participants were asked to recall their condition during the peak period of the first wave and to rate it using the same scale.

Results: The COS, PS, and K6 scores during the peak period were all significantly higher than those two months after the peak. There were positive correlations between $\mathrm{K} 6$ and COS scores and between $\mathrm{K} 6$ and PS scores during the peak period ( $r s=.574, P=.020$ and $r s=.587, P=.017$, respectively). However, these correlations were not significant two months after the peak. The correlation analysis including COS and K6 or PS showed that increased workload was positively correlated with K6 and PS scores during the peak period ( $\mathrm{r} s=.869, \mathrm{P}<.001 ; \mathrm{rs}=.519, \mathrm{P}=.040$, respectively). In the post-peak period, a positive correlation was found only between increased workload and $\mathrm{K} 6$ score ( $r \mathrm{~s}=.686, \mathrm{P}=.005)$. Insomnia was positively correlated with the $\mathrm{K} 6$ score both in the peak and post-peak periods ( $\mathrm{rs}=.656, \mathrm{P}=.006$ and $\mathrm{rs}=.565, \mathrm{P}=$. 028).

Conclusions: Among these Japanese nurses working in COVID-19 wards, COVID-19-related occupational stress, psychological distress, and physical symptoms were all decreased after the peak period compared with the peak period. During the peak period, associations were found between COVID-19-related occupational stress and psychological distress and between psychological distress and physical symptoms. Increased workload and insomnia were associated with psychological distress in both the peak and post-peak periods, and increased workload was associated with physical symptoms during the peak period.

\section{Background}

Since the outbreak of coronavirus disease 2019 (COVID-19) in China at the end of 2019, the global pandemic has created tremendous challenges for healthcare workers. COVID-19 quickly spread throughout Japan beginning in January 2020, sickening over 800,000 people and killing more than 15,000 as of July, 2021. To provide medical services with limited resources for the ever-increasing number of cases, healthcare workers are forced to work long hours and handle a heavy workload. Fear of 
COVID-19 infection has been reported to be a significant stressor. $(1-3)$ There is an urgent need to support the physical and mental health of medical personnel who work under these stressful conditions.

Reports on the mental health of healthcare workers treating COVID-19 patients have shown high levels of psychological distress, including anxiety and depression. (Lai J, 2020) A case-control study in China showed that frontline medical staff had more medical problems, such as anxiety symptoms, depressed mood, and insomnia, than non-frontline staff. (4) A study of medical staff in New York showed that psychological distress, such as acute stress, depressive symptoms, and anxiety symptoms, was increased, especially among nurses and advanced practice providers. (5) It is also well known that somatic complaints are associated with depression and anxiety. (6) A multicenter study of the psychiatric and somatic symptoms of medical staff during the COVID-19 outbreak reported that they exhibited somatic symptoms such as pain and insomnia. (7)

Thus, during the peak period of the first wave of the outbreak in Japan, COVID-19-related occupational stress, such as increased workload and anxiety about infection (hereinafter referred to as COVID-19related occupational stress) was reported to be increased and psychological distress and physical symptoms have been frequently reported. Because of the prolonged nature of the COVID-19 epidemic, it is important to understand changes over time in symptoms caused by COVID-19-related occupational stress and their associations in order to provide long-term support. However, to our knowledge no study has investigated change from the peak period to after the peak of the epidemic in terms of occupational stress, psychological distress, physical symptoms, and their correlations among Japanese frontline medical staffs. Our aim in doing this research was to clarify the impact of these variables so that we can design programs to ameliorate the effects of these stressors to improve the working conditions and support the mental health of frontline nurses.

Based on previous reports, we formulated and tested the following hypotheses. (1) COVID-19-related occupational stress, physical symptoms, and psychological distress would all improve after the peak period, and (2) there would be correlations among COVID-19-related occupational stress (fear of infection and increased workload), psychological distress, and physical symptoms (especially pain and insomnia) during the peak period.

\section{Methods}

\section{Participants}

Because previous studies on SARS and COVID-19 have shown that nurses are more likely to experience psychological distress than physicians because of their closer contact with patients $(8,9)$, we recruited nurses from the special unit for the care for patients with infectious diseases at the National Hospital Organization Fukuoka Higashi Medical Center, a designated medical institution for the treatment of infectious diseases. It is equipped with 23 negative pressure rooms, and we started care for COVID-19 
patients there in March 2020. The participants of this study included 16 frontline nurses (25-52 years old, 1 male) engaged in face-to-face care for COVID patients.

\section{Study design}

In our hospital, the peak of the first wave of COVID-19 admissions occurred in April and May of 2020 (T1), as shown in Fig. 1. We assessed COVID-19-related occupational stress, physical symptoms, and psychological distress among our frontline nurses as part of a mental support program during the period from June 23-28, 2020 (T2), two months after the first peak at a time when the number of patients had been greatly reduced. We explained the purpose of the survey, and all nurses agreed to participate. At that time, we asked them to both recall their status during the initial peak period ( $\mathrm{T} 1$ and to record their feelings at T2.

\section{Assessment items}

COVID-19-related occupational stress, somatic symptoms, and psychological distress were assessed using self-administered questionnaires about feelings over the past 30 days. Because at the time there were no standardized questionnaires specific to COVID-19, we developed two scales based on the items used in the Japanese government's stress check system for workers (10): a two-item COVID-19-related occupational stress scale and a four-item physical symptoms scale. In addition, the 6-item Kessler Scale (K6) score was used to assess psychological distress. (11) For all scales, respondents scored each item on a 5-point Likert scale of 0 (seldom) to 4 (very often), as in Table 1. 
Table 1

The questionnaire items used to assess occupational stress among frontline nurses working in a COVID19 ward.

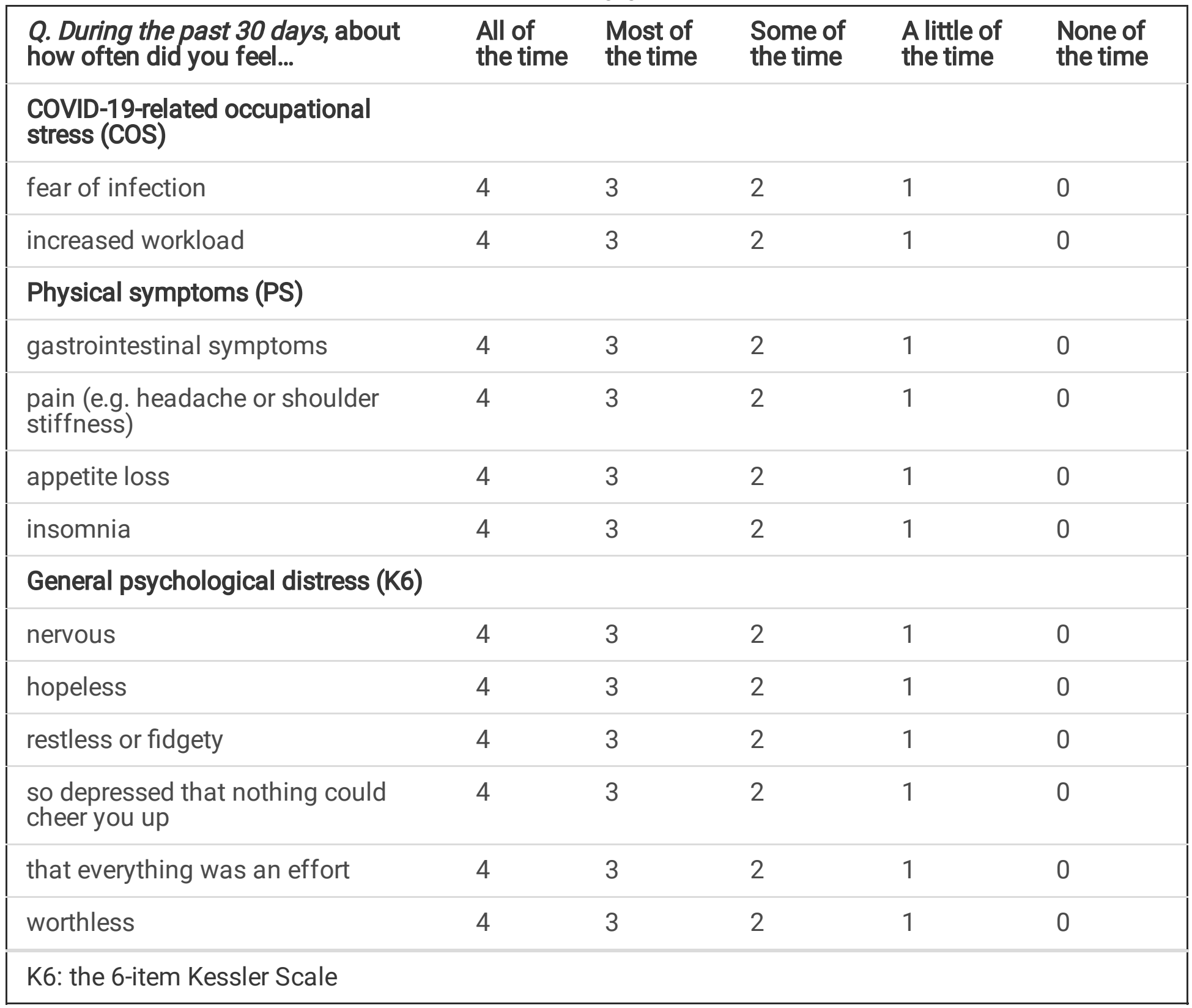

COVID-19-related occupational stress

Fear of infection and increased workload were selected as COVID-19-related occupational stress (COS) questionnaire items. It was expected that these stresses would increase during the peak period due to the unknown aspects of the situation and that these would be associated with physical symptoms and psychological distress.

Physical symptoms

The physical symptoms (PS) investigated included gastrointestinal symptoms, pain, appetite loss, and insomnia. If the patient had pain, the location of the pain was documented.

Psychological distress 
To assess psychological distress, we used the 6-item Kessler Scale (K6), a self-administered questionnaire that includes clinical depression and anxiety, including the following six items: nervousness, hopelessness, restlessness, sadness, unable to make efforts, and worthlessness. (12)

\section{Statistical analysis}

Statistical analysis was performed using the SPSS version $22.0 \mathrm{~J}$ statistical software package (IBM SPSS Statistics, Chicago, IL, USA). The Wilcoxon signed-rank test was applied to determine change in the variables from T1 to T2. Correlation analysis was performed using Spearman correlation analysis. The criterion for statistical significance was $\mathrm{P} \leq 0.05$.

\section{Results}

\section{Participant characteristics}

All participants completed the survey. The medians (lowest, 25th percentile - 75th percentile, highest) for age, nursing experience, and duration of assignment to a ward for infectious disease patients, including before the COVID-19 epidemic, were $30(25,26.75-43.75,56)$ years, $74(14,50-176,408)$ months, and 23 $(2,14.75-41,71)$ months, respectively.

\section{Changes in COVID-19-related occupational stress (COS), psychological distress (K6) and physical symptoms (PS)}

Figure 2A shows the changes in COS, PS, and $\mathrm{K} 6$ scales from T1 to T2. The T2 COS, PS, and K6 scores were significantly decreased compared with those of T1. The COS questionnaire items fear of infection and increased workload and the PS questionnaire items gastrointestinal symptoms, pain, appetite loss and insomnia were all significantly lower in the period after the peak (Fig. 2B).

\section{Prevalence of physical symptoms (PS) and their change}

In T1 among the four PS questions the prevalence of pain symptoms was the highest (68.8\%), followed by gastrointestinal symptoms $(62.5 \%)$, insomnia (56.3\%), and appetite loss $(50 \%)$. For those with pain, the pain location(s) were as follows: headache (54\%), back pain (27.3\%), and shoulder and neck pain (27.3\%).

In T2 the prevalence of the symptoms was pain (62.5\%), gastrointestinal symptoms (37.5\%), insomnia (43.8\%), and appetite loss (32.3\%).

\section{COVID-19 correlations with occupational stress (COS), psychological distress (K6) and physical symptoms (PS)}

To examine the association with COS, $\mathrm{K} 6$ and PS scores, correlation analysis between these scales was performed. At T1, positive correlations were found between COS and K6 scores and between K6 and PS scores ( $r s=.574, \mathrm{P}=.020 ; \mathrm{rs}=.587, \mathrm{P}=.017$, respectively). However, no significant correlation was found 
in $\mathrm{T} 2$ ( $\mathrm{rs}=.424, \mathrm{P}=.102 ; \mathrm{rs}=.441, \mathrm{P}=.088$, respectively). OS and PS scores showed no significant correlation in either $\mathrm{T} 1$ or $\mathrm{T} 2$ ( $\mathrm{T} 1 \mathrm{rs}=.100, \mathrm{P}=.712$; $\mathrm{T} 2 \mathrm{rs}=.142, \mathrm{P}=.600)$.

Correlation of increased workload and anxiety about infection with K6 and PS scores and pain and insomnia

The COS questionnaire items fear of infection and increased workload and the results of correlation of these items with $\mathrm{K} 6$ and PS scores and pain and insomnia, especially noting items in PS, are shown in Table 2. In T1, positive correlations were found between increased workload and $\mathrm{K} 6$ scores and between increased workload and PS scores ( $r s=.869, \mathrm{P}<.001$ and $\mathrm{rs}=.519, \mathrm{P}<.05$, respectively). However, in T2 a positive correlation was shown only between increased workload and $K 6$ scores $(r s=.686, P<.01)$. There was no significant correlation between fear of contagion and $\mathrm{K} 6$ scores or between fear of contagion and PS scores in either T1 or T2.

\section{Table 2}

Correlations.

Correlations of COVID-19-related occupational stress (COS) (increased workload and fear of contagion), general psychological distress (K6), and two physical symptom items (PS) (insomnia and pain) at T1 and

T2. The data are shown as R-value (correlation coefficient; rs) and significance level $\left({ }^{*} P<0.05, * \star P<\right.$ $0.01)$.

\begin{tabular}{|c|c|c|c|c|c|}
\hline & & K6 & PS & insomnia & pain \\
\hline \multirow[t]{2}{*}{$\begin{array}{l}\text { Increased } \\
\text { workload }\end{array}$} & $\mathrm{T} 1$ & $\begin{array}{l}\mathrm{rs}=.869 * * \mathrm{p} \\
<.001\end{array}$ & $\begin{array}{l}r s=.519 * p \\
=.040\end{array}$ & $\begin{array}{l}r s=.498 * p \\
=.050\end{array}$ & $\begin{array}{l}r s=.490 p \\
=.054\end{array}$ \\
\hline & $\mathrm{T} 2$ & $\begin{array}{l}\mathrm{rs}=.732^{* *} \mathrm{p} \\
<.001\end{array}$ & $\begin{array}{l}r s=.219 p \\
=.415\end{array}$ & $\begin{array}{l}r s=.373 p \\
=.155\end{array}$ & $\begin{array}{l}r s=.192 p \\
=.476\end{array}$ \\
\hline \multirow[t]{2}{*}{ Fear of infection } & $\mathrm{T} 1$ & $\begin{array}{l}r s=.205 p \\
=.447\end{array}$ & $\begin{array}{l}r s=-.209 p \\
=.438\end{array}$ & $\begin{array}{l}r s=-.238 p \\
=.375\end{array}$ & $\begin{array}{l}\mathrm{rs}=-.166 \mathrm{p} \\
=.538\end{array}$ \\
\hline & $\mathrm{T} 2$ & $\begin{array}{l}r s=.036 p \\
=.882\end{array}$ & $\begin{array}{l}r s=-.069 p \\
=.800\end{array}$ & $\begin{array}{l}r s=-.024 p \\
=.930\end{array}$ & $\begin{array}{l}r s=-.161 p \\
=.552\end{array}$ \\
\hline \multirow[t]{2}{*}{ K6 } & $\mathrm{T} 1$ & - & $\begin{array}{l}r s=.587 * p \\
=.017\end{array}$ & $\begin{array}{l}r s=.656 * * p \\
=.006\end{array}$ & $\begin{array}{l}r s=.492 p \\
=.053\end{array}$ \\
\hline & $\mathrm{T} 2$ & - & $\begin{array}{l}r s=.441 p \\
=.088\end{array}$ & $\begin{array}{l}r s=.589 * p \\
=.016\end{array}$ & $\begin{array}{l}r s=.428 p \\
=.098\end{array}$ \\
\hline
\end{tabular}

A positive correlation was found between increased workload and insomnia score only in T1 ( $r s=.498, p$ $<0.05)$. No significant correlation was found between anxiety of infection and pain or insomnia in either $\mathrm{T} 1$ or $\mathrm{T} 2$. There was no correlation between pain and $\mathrm{K} 6$ scores in either $\mathrm{T} 1 \mathrm{or} \mathrm{T} 2$; however, there was a positive correlation between insomnia and $\mathrm{K} 6$ scores in both $\mathrm{T} 1$ and $\mathrm{T} 2$ ( $\mathrm{rs}=.656, \mathrm{P}=.006$ and $\mathrm{rs}=.656$, $\mathrm{P}=.006$, and $\mathrm{rs}=.565, \mathrm{P}=.028$, respectively).

\section{Discussion}


Our survey of Japanese nurses working in inpatient wards for COVID-19 patients found that COVID-19related occupational stress, physical symptoms, and psychological distress were high during the peak period of the first wave, then decreased after the peak had passed. Correlations were found between COVID-19-related occupational stress and psychological distress, and between psychological distress and physical symptoms during the peak period, but not two months after the peak. Notably, increased workload and insomnia were associated with psychological distress regardless of the peak or post-peak period, and increased workload was also associated with physical symptoms during the peak period. The present study is the first Japanese report to show an interrelation between COVID-19-related occupational stress, psychological distress, and physical symptoms.

\section{Changes in COVID-19-related occupational stress, psychological distress, and physical symptoms}

Previous studies have reported occupational stress, such as increased workload, fear of infection, physical symptoms such as pain and insomnia, and psychological distress among HCWs during the COVID-19 pandemic. $(7,9,13)$ Their results were confirmed for Japanese HCWs in the present study. In addition, our finding that COVID-19-related occupational stress, psychological distress, and physical symptoms during the peak period were improved two months after the peak suggests that some of these mental health problems are reversible. However, if the outbreak is repeated in future waves and the period of engagement is prolonged, the results might be different. Future cross-sectional follow-up studies on changes during and after repeated waves will be important.

\section{Prevalence of physical symptoms and their changes}

Among the somatic symptoms, the prevalence of insomnia (56.3\%) in our study was higher than that seen in in previous reports, which ranged from $21-39.3 \% .(7,9,13)$ This difference may be related to the high prevalence of insomnia reported in Japan and the exacerbation of insomnia due to the increased workload, as discussed below. Furihata reported that $41.4 \%$ of female hospital nurses in Japan presented short sleep duration, less than six hours. (14) Because of the high prevalence of insomnia in Japanese healthcare workers, programs should be implemented to follow them carefully and to provide intervention where necessary.

\section{Interrelation between occupational stress, psychological distress, and physical symptoms}

We revealed for the first time an association between COVID-19-related stress and physical symptoms among Japanese healthcare workers treating COVID-19 during the peak infection period. A previous report showed that occupational stressors (nursing workload and fear of infection) were associated with high risk of anxiety and depressive symptoms (15). An association between occupational stress and psychological distress was also found in the present study, however, there was no association between anxiety about infection and physical symptoms related to COVID-19. These differences may be due to differences in the subjects and/or questionnaires. 
Previous studies have reported that appetite loss was correlated with depression and anxiety. (7) In the current study, the total score of physical symptoms (gastrointestinal symptoms, pain, appetite loss and insomnia) was positively correlated with $\mathrm{K} 6$. This result suggests that it is necessary not only to pay attention to psychological distress but also to these physical symptoms during future waves of this or other infectious events. Although the post-peak OS, PS, and K6 scores were not correlated, it is possible that the decrease in the values of OS, PS, and K6 scores might be related to these results.

Possible interrelations were analyzed with respect to increased workload and fear of infection in OS and for pain and insomnia in PS. The results showed increased workload and insomnia to be associated with psychological distress regardless of peak or post-peak period and that increased workload was associated with insomnia both during and after the peak period. It has been reported that "few non-work days" is a risk factor for increased PHQ-9 (Patient Health Questionnaire-9; self-administered depression scale) scores among Japanese hospital workers treating COVID-19 (16). Therefore, the increased workload and fewer non-work days may have caused exacerbation of psychological distress such as depression and physical symptoms including insomnia in Japanese medical personnel during the peak period.

Pain was not associated with $\mathrm{K} 6$ in either the peak or post-peak period, which is incompatible with our hypothesis on pain. From the perspective of conceivable courses of pain, because it might take a longer time for pain to become symptomatic, future longitudinal studies will needed to clarify the association with pain.

Previous reports and our present results suggest that to reduce psychological distress during the peak periods of the COVID-19 outbreak, it is important to reduce COVID-19-related occupational stress, especially to prevent an increase in workload and not to reduce non-work days. Of the physical symptoms, it is important to pay careful attention to insomnia.

\section{Limitations}

There are several limitations to our study. First, the number of participants was small. Therefore, we are currently conducting a prospective study with a larger number of participants. Also, the present study lacks data on baseline values, including history of physical symptoms. However, because OS, PS, and K6 scores all declined after the peak, it is thought that the baseline scores would be similar to or lower than the post-peak scores. Furthermore, we used retrospective questionnaires in part of the study, which has the problem of recall bias. This issue needs to be addressed in future prospective studies. Finally, the participants were overwhelmingly female, however, the proportion is similar to the overall proportion of nurses in Japan (7.8\% males) (Report on Public Health Administration and Services in Japan, 2018). Therefore, we considered the study to be representative of the nurses working in COVID-19 wards in Japan.

\section{Conclusion}


The occupational stress, physical symptoms and psychological distress of our participating nurses working in a ward for COVID-19 patients decreased after the peak period of the first wave of COVID-19 infection. During the peak period, there were associations between COVID-19-related occupational stress and psychological distress and between psychological distress and somatic symptoms. An increased workload and insomnia were associated with psychological distress regardless of the period, and increased workload was associated with physical symptoms during only the peak period.

\section{Abbreviations}

COVID-19: coronavirus disease 2019

K6: the 6-item Kessler Scale

\section{Declarations}

\section{Ethics approval and consent to participate}

This study was approved by the Clinical Research Ethics Committee of the National Hospital Organization Fukuoka Higashi Medical Center (Ref. No.2021-C-12).

\section{Consent for publication}

Not applicable.

\section{Availability of data and materials}

Not applicable.

\section{Competing interests}

The authors declare that they have no competing interests.

\section{Funding}

Not applicable.

\section{Authors' contributions}

TN designed and carried out the survey. AO wasa major contributor in planning and conducting the survey. TN\&MK conceived the data and wrote the manuscript. TN\&KYinterpreted data, considered statistical calculations, and structured the report. KY supervised and corrected the manuscript.All authors have read and approved the final manuscript.

\section{Acknowledgements}


We thank the participants for their cooperation.

\section{References}

1. Tucci V, Moukaddam N, Meadows J, Shah S, Galwankar SC, Kapur GB. The Forgotten Plague: Psychiatric Manifestations of Ebola, Zika, and Emerging Infectious Diseases. J Glob Infect Dis. 2017;9:151-156. doi:10.4103/jgid.jgid_66_17.

2. Maunder R, Hunter J, Vincent L, Bennett J, Peladeau N, Leszcz M, et al. The immediate psychological and occupational impact of the 2003 SARS outbreak in a teaching hospital.CMAJ. 2003;168:124551.

3. Wu P, Fang Y, Guan Z, Fan B, Kong J, Yao Z, et al. The psychological impact of the SARS epidemic on hospital employees in China: exposure, risk perception, and altruistic acceptance of risk.Can $\mathrm{J}$ Psychiatry. 2009;54:302-11.doi: 10.1177/070674370905400504.

4. Cai Q, Feng H, Huang J, Wang M, Wang Q, Lu X, et al. The mental health of frontline and non-frontline medical workers during the coronavirus disease 2019 (COVID-19) outbreak in China: A case-control study. J Affect Disord. 2020;275:210-215.doi: 10.1016/j.jad.2020.06.031.

5. Shechter A, Diaz F, Moise N, Anstey DE, Ye S, Agarwal S, et al. Psychological distress, coping behaviors, and preferences for support among New York healthcare workers during the COVID-19 pandemic. Gen Hosp Psychiatry. 2020;66:1-8. doi: 10.1016/j.genhosppsych.2020.06.007.

6. Kroenke K, Jackson JL, Chamberlin J. Depressive and anxiety disorders in patients presenting with physical complaints: clinical predictors and outcome. Am J Med. 1997;103:339-47. doi:

10.1016/s0002-9343(97)00241-6.

7. Chew NWS, Lee GKH, Tan BYQ, Jing M, Goh Y, Ngiam NJH, et al. Amultinational, multicentre study on the psychological outcomes and associated physical symptoms amongst healthcare workers during COVID-19 outbreak. Brain Behav Immun. 2020;88:559-565. doi: 10.1016/j.bbi.2020.04.049.

8. Lai J, Ma S, Wang Y, Cai Z, Hu J, Wei N, et al. Factors Associated With Mental Health Outcomes Among Health Care Workers Exposed to Coronavirus Disease 2019. JAMA Netw Open. 2020;3:e203976. doi: 10.1001/jamanetworkopen.2020.3976.

9. Wong TW, Yau JK, Chan CL, Kwong RS, Ho SM, Lau CC, et al. The psychological impact of severe acute respiratory syndrome outbreak on healthcare workers in emergency departments and how they cope. Eur J Emerg Med. 2005;12:13-8. doi: 10.1097/00063110-200502000-00005.

10. Kawakami N, Tsutsumi A. The Stress Check Program: a new national policy for monitoring and screening psychosocial stress in the workplace in Japan. J Occup Health. 2016;58:1-6. doi: 10.1539/joh.15-0001-ER.

11. Kessler RC, Andrews G, Colpe LJ, Hiripi E, Mroczek DK, Normand SL, et al.Short screening scales to monitor population prevalences and trends in non-specific psychological distress. PsycholMed. 2002;32:959-976. doi: 10.1017/S0033291702006074. 
12. Kessler RC, Barker PR, Colpe LJ, Epstein JF, Gfroerer JC, Hiripi E, et al. Screening for serious mental illness in the general population. Arch Gen Psychiatry. 2003;60:184-189. doi: 10.1001/archpsyc.60.2.184.

13. Zhang WR, Wang K, Yin L, Zhao WF, Xue Q, Peng M, et al. Mental Health and Psychosocial Problems of Medical Health Workers during the COVID-19 Epidemic in China. PsychotherPsychosom. 2020;89:242-250. doi: 10.1159/000507639.

14. Furihata R, Saitoh K, Otsuki R, Murata S, Suzuki M, Jike M, et al. Association between reduced serum BDNF levels and insomnia with short sleep duration among female hospital nurses. Sleep Med. 2020;68:167-172. doi: 10.1016/j.sleep.2019.12.011.

15. Zheng R, Zhou Y, Fu Y, Xiang Q, Cheng F, Chen H, et al.Prevalence and associated factors of depression and anxiety among nurses during the outbreak of COVID-19 in China: A cross-sectional study. Int J Nurs Stud. 2021;114:103809. doi: 10.1016/j.jnurstu.2020.103809.

16. Matsumoto Y, Fujino J, Shiwaku H, Miyajima M, Doi S, Hirai N, et al. Factors affecting mental illness and social stress in hospital workers treating COVID-19: Paradoxical distress during pandemic era. J Psychiatr Res. 2021;137:298-302. doi: 10.1016/j.jpsychires.2021.03.007

\section{Figures}

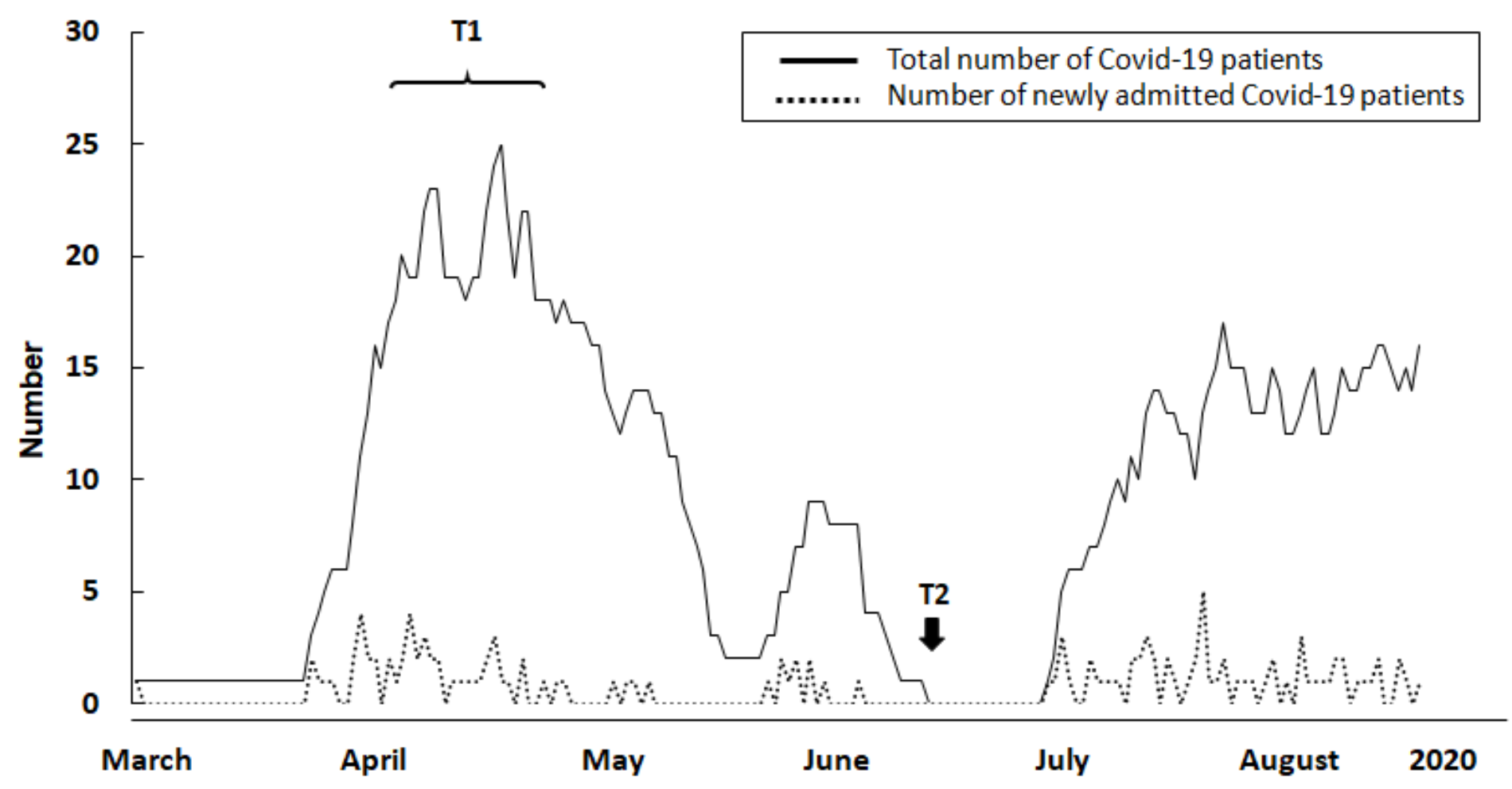

\section{Figure 1}

Change in the total number and number of newly admitted patients with COVID-19. The peak of the first COVID-19 epidemic occurred in April and May of 2020 (T1) in National Hospital Organization Fukuoka 
Higashi Medical Center. COVID-19-related occupational stress, physical symptoms, and psychological distress were assessed for frontline nurses as part of a mental support program done from June 23-28, 2020 (T2), two months after the peak of the first COVID-19 wave. The solid line and dotted line show the total number of COVID-19 patients hospitalized and the number of newly admitted patients, respectively.

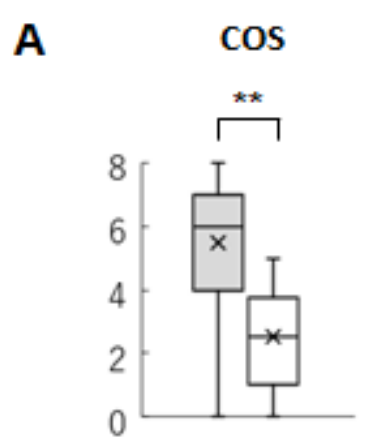

T1 T2

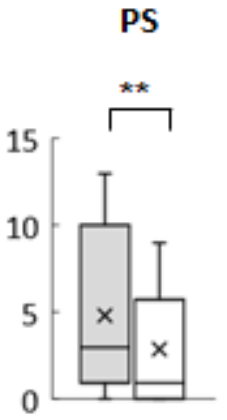

T1 T2
K6

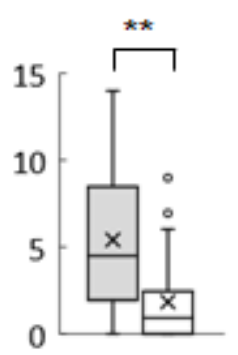

T1 T2

B

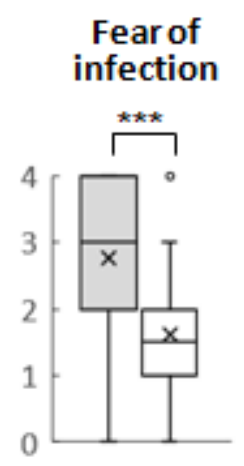

T1 T2

\section{Increased workload}

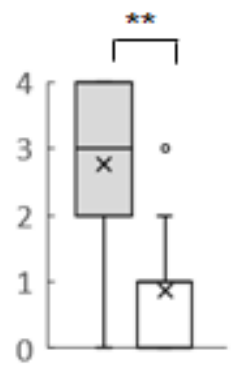

T1 T2
GI symptom

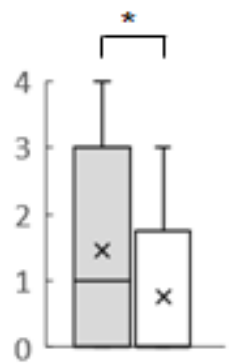

T1 T2
Pain

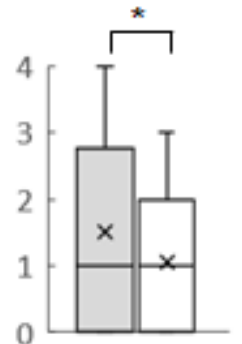

T1 T2

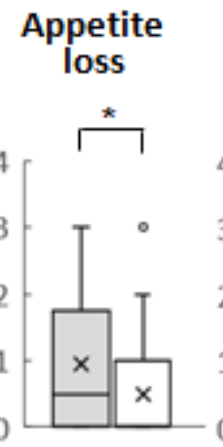

T1 T2
Insomnia

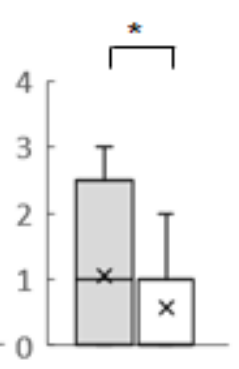

T1 T2

\section{Figure 2}

Change from T1 to T2 in COVID-19-related occupational stress, physical symptoms, and 6-item Kessler Scale. $\triangle A$ )The T2 scores for COVID-19-related occupational stress (COS), physical symptoms (PS), and the 6-item Kessler Scale (K6) scores were significantly decreased from those of T1. (B) Fear of infection and increased workload of COS and gastrointestinal (GI) symptoms, pain, appetite loss, and insomnia of PS significantly decreased after the peak period. The data are shown using box-and-whisker plots. The whiskers mark the 5th and 95th percentiles, with the upper and lower end of the boxes showing the third and first quartile ranges of the data, respectively. Median values are expressed as the line inside the box. An asterisk indicates a significant difference $\left(*: p<.05,{ }^{* \star}: p<.01,{ }^{\star \star *}: p<.001\right)$. 